\title{
Las representaciones sociales del alcoholismo: una perspectiva de género
}

\author{
Eugenia Galicia González*, Marco Polo García Durán** \\ Juan José Monroy Ramirez***, Julio César Navarro Becerril**** \\ Asesoras: Mtra. Silvia Gutiérrez Vidrio \\ y Mtra. Ma. del Carmen de Lara Rangel \\ Universidad Autónoma Metropolitana, \\ UNIDAD XOCHIMILCO
}

Hoy en día es difícil imaginar una sociedad ajena a los problemas que causan al ser humano los productos nocivos para la salud como son el alcohol, el tabaco y algunas drogas o sustancias que alteran el sistema nervioso, ya que en cualquier lugar podemos encontrar publicidad o actividades en las que el consumo de estos productos está presente.

El consumo de alcohol tiene lugar dentro de la mayoría de los ámbitos sociales como: la familia, el trabajo, la escuela y situaciones diversas como celebraciones y ceremonias; ejemplos existen bastantes: bodas, bautizos, fiestas religiosas, partidos de fútbol, etcétera; así mismo las motivaciones por las cuales es consumido son distintas: tristeza, felicidad, depresión, nostalgia, en fin, siempre hay un motivo que lleva al ser humano a beber sustancias etílicas.

\footnotetext{
* Licenciada en Comunicación Social, Universidad Autónoma Metropolitana, Unidad Xochimilco. Ha demostrado especial interés por las áreas de producción y realización audiovisual, periodismo, y elaboración de artículos informativos y de difusión. Obtuvo Mención Honorífica por el documental Medias de Seda en el Segundo Festival de Cortometraje El cine a las calles en 2006, entre otros. ** Licenciado en Comunicación Social, Universidad Autónoma Metropolitana, Unidad Xochimilco. Ha incursionado en diversas actividades de fotografía; proyectos de radio y televisión de la UAM, Unidad Xochimilco; así como proyectos de cine y video, entre otros.

*** Licenciado en Comunicación Social, Universidad Autónoma Metropolitana, Unidad Xochimilco. Ha incursionado en diversos proyectos la UAM, Unidad Xochimilco, así como ha asistido proyectos con el Centro de Capacitación Cinematográfica, y laborado como freelance en empresas productoras como The Pacific Group, Rojo Films, Agave Films, y un programa piloto con Televisa. Ha recibido menciones honoríficas como correalizador de documentales en el 20 Festival de cortometrajes El cine a las Calles, y el Concurso Las mujeres desde los ojos de la Juventud, organizado por el IMJUvE.

**** Licenciado en Comunicación Social, Universidad Autónoma Metropolitana, Unidad Xochimilco.
} 
No es erróneo pensar que en casi todas las sociedades del mundo, y más específicamente en la sociedad mexicana, el consumo de alcohol esté ligado a las actividades festivas que día con día se celebran en el país, pero sobre todo juega un papel importante como elemento de socialización. El beber alcohol, como menciona Hugo Hiriart, cumple funciones múltiples en las sociedades como medio de interacción a nivel social, económico y cultural (1987, p. 11), no en vano las grandes compañías cerveceras y licoreras invierten cantidades exorbitantes de dinero en publicidad para lograr un consumo masivo de sus productos por la mayoría de la población.

Sin embargo, este consumo puede convertirse en adicción cuando se pierde el control en la manera de beber. En este trabajo nos referiremos al alcoholismo como: el consumo de bebidas alcohólicas que en exceso repercuten en las relaciones sociales para convertirse en un problema; esta adicción conlleva una dependencia física y psicológica del alcohol, es decir, en una necesidad o deseo compulsivo por beber.

Las consecuencias que enfrentan las personas que padecen esta enfermedad son múltiples, sufren graves problemas de salud, de integración social, ya que la sociedad los margina y excluye de los grupos sociales, y problemas psicológicos como depresión y neurosis debido al rechazo que sufren por los individuos que les rodean.

El rechazo y marginación de las personas que padecen alcoholismo está relacionado con la edad, la condición social, la comunidad con la que se relacionan y también al género al que pertenecen. En nuestro estudio se retoma la perspectiva de género porque consideramos que es a través del mismo, que la sociedad construye la imagen del hombre y la mujer a partir de sus diferencias biológicas, que orientan su comportamiento dentro de la sociedad. En cierto sentido, los niveles de jerarquía en una sociedad se definen de acuerdo con la cuestión de género; en palabras de Marta Lamas: “...el género es un elemento constitutivo de las relaciones sociales [...] y es una forma primaria de relaciones significantes de poder" (1996, p. 331). Es por eso que no es de extrañarse que en las actividades donde predominan los hombres, la mujer sea mal vista o criticada si se encuentra realizando funciones o labores que en un principio pueden entenderse como pertenecientes al género masculino.

En el caso del consumo de alcohol, al ser un producto consumido tanto por mujeres como por hombres se podría pensar que la tolerancia social que se tiene respecto a esta práctica es la misma para los dos géneros; sin embargo dicho consumo, en la mayoría de las veces, está ligado al estereotipo masculi- 
no (que califica al hombre como fuerte, valiente, dominante), así que cuando una mujer bebe se le critica porque está haciendo algo que no es acorde con la imagen de la mujer ideal (sensible, callada, dedicada al hogar, maternal, débil) que la sociedad ha construido de ella, por lo que si una mujer padece alcoholismo, la marginación y desaprobación por parte de la sociedad es mayor. Si bien el rechazo hacia los alcohólicos es inminente, a la mujer se le margina por ser alcohólica y al mismo tiempo por ser mujer, ya que rompe con la imagen de la mujer tradicional, estereotipo implantado por la misma sociedad en la que se desenvuelve. Esto ocasiona en las mujeres una baja en su autoestima y en las relaciones con su familia y con las personas del entorno en el que se desarrolla.

Los estudios de género entienden al sexo como un sistema socialmente prescripto que determina los comportamientos en la sociedad, por la diferencia que establece ser hombre o ser mujer. La sociedad tiende a decidir las actividades y actitudes que deben realizar las personas en relación a su condición de hombre o mujer de acuerdo con la representación que de ellas se ha formado. En el caso del alcoholismo también existe la posibilidad de que las personas intenten recuperar lo que debido a su forma de beber, vieron afectado como son: el amor hacia su persona, la pérdida del lazo familiar, o las relaciones sociales; esta posibilidad se logra a través de un tratamiento de recuperación. Es por eso que en esta investigación lo que nos interesa abordar es la representación social del alcoholismo desde la percepción de mujeres alcohólicas en recuperación.

La representación social es un sistema de valores, ideas y prácticas que tiene una doble función: poner un orden que permita a los individuos orientarse en su mundo material social y posibilitar la comunicación entre los miembros de una comunidad, proporcionándoles un código de intercambio social y un código para nombrar y clasificar los fenómenos sociales (Moscovici, 1979, p. 17).

Este conocimiento, como menciona Jodelet, se constituye a partir de nuestras experiencias, pero también de las informaciones, conocimientos y modelos de pensamiento que recibimos y transmitimos a través de la tradición, la educación y la comunicación social (1986, p. 473).

En este sentido y considerando las particularidades sociales antes mencionadas, esta investigación trata de dar respuesta a las siguientes interrogantes, ¿cuál es la representación social que las mujeres alcohólicas en recuperación tienen acerca del alcoholismo?, ¿de qué manera afecta la cuestión de género en el alcoholismo de una persona?, ¿se vive de la misma forma el alcoholismo en una mujer que en un hombre? 
El interés que motivó esta investigación es el hecho de que la problemática del alcoholismo es tolerada de forma distinta por la sociedad, a consecuencia de la construcción social del género que se tiene en México. Es por eso que el objetivo central es conocer la representación social que un grupo de mujeres en recuperación tiene acerca del alcoholismo por medio de sus experiencias de vida, ya que al haber padecido el problema tienen una visión particular acerca del consumo excesivo de alcohol.

La particularidad de la investigación radica en la perspectiva metodológica utilizada para captar el punto de vista de los actores, lo que de cierto modo hace aún más comprensible la problemática a la que se enfrentan las mujeres alcohólicas en una sociedad machista como es la mexicana. Dos hipótesis guían la investigación: 1) La percepción del alcoholismo en una mujer es diferente a la del hombre debido a que la tolerancia en el consumo de alcohol en hombres es distinta a la que las mujeres enfrentan; y 2) La sociedad tolera, rechaza, y juzga de manera diferente el alcoholismo de un hombre y el de una mujer, debido a la imagen construida socialmente para cada género.

Para abordar la problemática de las representaciones sociales existen diferentes métodos. En nuestro caso elegimos el método cualitativo, que permite captar el proceso de construcción de las representaciones sociales. El instrumento de recopilación de información que se privilegió fue la entrevista focalizada, ya que es a través de ésta que pudimos obtener información que nos sirviera para identificar las diferencias y similitudes en la conceptualización de la problemática en cada una de las informantes. Se realizaron entrevistas a un grupo de ocho mujeres alcohólicas en recuperación de diferente estado civil y nivel socioeconómico, lo que nos permitió obtener información de distintos estratos de la sociedad.

A partir de la información obtenida se llevó a cabo el análisis del discurso de las entrevistas. Por medio de la propuesta del análisis argumentativo se identificaron los principales tópicos y subtópicos que aparecen en el corpus de estudio. Cada tópico o dimensión es ilustrada con los testimonios que más claramente expresan las opiniones de las informantes sobre el tema. Posteriormente se realizó la interpretación de los testimonios y se llegó a las siguientes conclusiones:

El alcoholismo además de ser una adicción es también la reproducción de una acción, es decir, en la mayoría de los casos se debe en gran medida a la reproducción de patrones de conducta en la sociedad donde se desarrolla, en esta época es perceptible el observar a gente bebiendo, pareciera que es algo muy obvio pero la realidad es que el consumo de alcohol se ha convertido en 
una actividad tan común en la vida diaria que ahora es visto por la sociedad como una actividad que pareciera que no tiene consecuencias graves.

En el ámbito emocional, la mujer padece de manera distinta el alcoholismo ya que debido a los preconstruidos culturales que se tienen acerca de lo que es "ser mujer" siente aflicción y remordimientos consigo misma por no poder actuar y comportarse de acuerdo a los patrones socialmente establecidos.

En lo que concierne al ámbito social, es difícil encontrar personas que juzguen el alcoholismo de una mujer de la misma manera que en un hombre, porque ha sido la sociedad la principal responsable de esta falta de aceptación, causado por la imagen que se ha construido de las personas del sexo femenino como: sumisas, dedicadas al hogar, dependientes y correctas, que contrasta con la imagen que la sociedad ha creado de la persona alcohólica como un individuo irresponsable, carente de valores, poco confiable y vulgar.

Además, la mujer alcohólica se enfrenta a una lucha psicológica cuando se integra a sus actividades normales y al mismo tiempo continúa en el grupo de estigmatizados al que pertenece, ya que representa un obstáculo el convivir con la sociedad mientras pertenece a un grupo estigmatizado como lo es Alcohólicos Anónimos. No es fácil para un alcohólico y mucho menos lo es para una mujer alcohólica, enfrentarse como lo hemos observado, ante la propia familia y la sociedad. La complejidad del problema radica en el lugar que ocupa la mujer en la sociedad, por lo que al incurrir en este tipo de conductas desviantes es juzgada más duramente por todo lo que se espera de ella.

En relación a la identificación de la representación social de las mujeres alcohólicas acerca del alcoholismo, se hizo en dos etapas: 1) la representación social que construyeron antes del tratamiento de recuperación y 2) después del tratamiento de recuperación.

La representación social que las informantes tenían acerca del alcoholismo antes de entrar en proceso de recuperación era que el consumo de alcohol servía como un desinhibidor que les permitía desenvolverse socialmente con mayor facilidad. Desconocían totalmente la posibilidad de que este consumo les generara una dependencia adictiva así como las consecuencias físicas, psicológicas y sociales que les podía causar el exceso en el consumo. Además, para las informantes el ser alcohólica era improbable, ya que para ellas el ser alcohólica significaba "estar tirada en la calle", "ser una teporocha", "ser una mujer vulgar y que ha perdido sus valores". En lo que se refiere a los grupos de alcohólicos anónimos, las entrevistadas creían que eran lugares a los que llegaba "la gente del estrato social más bajo", "la peor gente", a donde ellas nunca podrían llegar. 
Por otro lado, en lo que concierne a la percepción que tienen las informantes ahora que llevan a cabo un tratamiento de recuperación es totalmente distinta, ya que debido al tratamiento han aprendido, de acuerdo con la doctrina de Alcohólicos Anónimos, que el alcoholismo es una enfermedad progresiva, degenerativa y de consecuencias fatales. Además, ahora consideran que el alcoholismo puede padecerlo cualquier persona, sin importar la edad, la condición económica o el sexo.

Por último, podemos concluir que: la imagen que ha construido la sociedad de las mujeres alcohólicas, estén o no en proceso de recuperación, es de una persona con falta de carácter y que ha perdido sus valores morales a consecuencia del consumo excesivo de alcohol, lo que ocasiona que los abusos a su personas lleguen a convertirse en derivaciones de su enfermedad.

Esta investigación sirvió para entender que en la sociedad mexicana, a pesar de que hoy en día la mujer ha podido desprenderse de muchos prejuicios y estereotipos negativos, sigue prevaleciendo una desaprobación en la imagen de la mujer alcohólica sin comprender que el alcoholismo es una problemática cada vez más preocupante, ya que atraviesa por todos los estratos sociales, edades, géneros o religiones.

\section{REFERENCIAS}

Hiriart, H. (1987). Vivir y Beber. México: Ediciones Cal y Arena.

Jodelet, D. (1986). "La representación social: fenómenos conceptos y teoría". En S. Moscovici Psicología Social II. Barcelona: Paidós. pp. 469-494.

Lamas, M. (1996). (comp.). "Usos, dificultades y posibilidades de la categoría <género>”. En El género: la construcción cultural de la diferencia sexual. México: PUEG-UnAm/ Porrúa. pp. 327- 376.

Moscovici, S. (1979). El psicoanálisis, su imagen y su público. Buenos Aires: Editorial Huemul. pp. 11-137.

$360 \cdot$ Resúmenes de tesis 